\title{
Transdermal Bioavailability in Rats of Lidocaine in the Forms of Ionic Liquids, Salts, and Deep Eutectic
}

\author{
Paula Berton, ${ }^{\dagger, \ddagger}$ Kristin R. Di Bona, ${ }^{\dagger, \S, \#}$ Denise Yancey, ${ }^{\dagger}$ Syed A. A. Rizvi, ${ }^{\prime \prime}$ Marquita Gray, ${ }^{\S}$ \\ Gabriela Gurau, ${ }^{\ddagger}$ Julia L. Shamshina, ${ }^{\ddagger} \perp$ Jane F. Rasco, ${ }^{\S}$ and Robin D. Rogers, \\ ${ }^{\dagger}$ Department of Chemistry, The University of Alabama, Tuscaloosa, Alabama 35487, United States \\ ${ }^{\ddagger}$ Department of Chemistry, McGill University, 801 Sherbrooke Street West, Montreal, Quebec H3A 0B8, Canada \\ ${ }^{\S}$ Department of Biological Sciences, The University of Alabama, Tuscaloosa, Alabama 35487, United States \\ "Department of Pharmaceutical Sciences, College of Pharmacy, Nova Southeastern University, Fort Lauderdale, Florida 33328, \\ United States \\ ${ }^{\perp} 525$ Solutions, Inc., 720 2nd Street, Tuscaloosa, Alabama 35401, United States
}

\section{Supporting Information}

\begin{abstract}
Tuning the bioavailability of lidocaine was explored by its incorporation into the ionic liquid lidocainium docusate $([\mathrm{Lid}][\mathrm{Doc}])$ and the deep eutectic Lidocaine-Ibuprofen (Lid-Ibu) and comparing the transdermal absorption of these with the crystalline salt lidocainium chloride $([\mathrm{Lid}] \mathrm{Cl})$. Each form of lidocaine was dissolved in a vehicle cream and topically applied to Sprague-Dawley rats. The concentrations of the active pharmaceutical ingredients (APIs) in blood plasma were monitored over time as an indication of systemic absorption. The concentration of lidocaine in plasma varied between applied API-based creams, with faster and higher systemic absorption of the hydrogen bonded deep eutectic Lid. Ibu than the absorption of the salts [Lid]Cl or [Lid][Doc]. Interestingly, a differential transdermal absorption was observed between lidocaine and ibuprofen when Lid-Ibu was applied, possibly indicating different interactions with the tissue components.
\end{abstract}

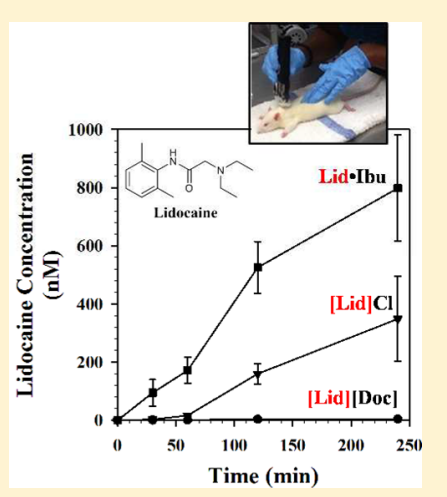

KEYWORDS: Active pharmaceutical ingredients, ionic liquids, deep eutectic, lidocaine, ibuprofen, docusate, bioavailability

\begin{abstract}
A ctive pharmaceutical ingredient-ionic liquids (API-ILs) have been reported and promoted as liquid forms of the active substances in conventional pharmaceutical solids which overcome many problems observed with crystalline pharmaceuticals, e.g., polymorphism and limited bioavailability. ${ }^{1,2}$ The molar ratio between the API and counterion and the degree of ionicity are critical for the properties relevant for the pharmaceutical industry since these influence bioavailability, including solubility, absorption, distribution, metabolism, and excretion. ${ }^{3}$ However, although it was initially suggested that APIILs were composed entirely of dissociated ions, spectroscopic data showed that there is a wide range in the degree of ionicity in these liquid forms, including not only ionic liquids (ILs) but also oligomeric ILs and deep eutectics, which have been termed liquid cocrystals. $^{3-5}$ An incomplete characterization and misunderstanding of the interactions between the components could interfere with their classification, as well as the anticipated mechanism of action of these forms. A clear example of this is the compound formed by mixing lidocaine and ibuprofen (Lid·Ibu), which has been defined in some publications as a true API-IL (completely ionized), ${ }^{6}$ though a comprehensive analysis has demonstrated that these ions form a deep eutectic liquid cocrystal via strong hydrogen bonds or interactions between partially ionized species (Figure 1$)^{7}$
\end{abstract}

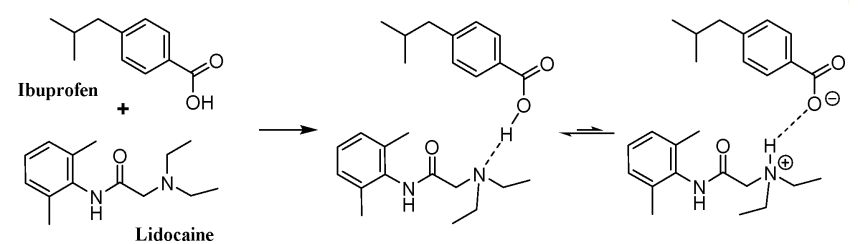

Figure 1. Interactions between lidocaine and ibuprofen in the liquid cocrystal Lid·Ibu.

Different biological behavior is expected from the API-based compounds depending on these interactions. For example, in compounds defined as salts, either solids or liquids, the ions are typically independent, and the bioavailability is expected to be lower since the membrane transport of ionized compounds is limited. ${ }^{8}$ This is not the case when the components are forming a hydrogen-bonded complex, ${ }^{8}$ e.g., in Lid·Ibu. When Lid·Ibu is dissolved in ethanol, the two actives are held together via strong hydrogen bond interactions and simultaneously transported through a model silicone membrane, with almost identical transport rates of both APIs, and faster than the API in solutions

Received: December 12, 2016

Accepted: April 12, 2017

Published: April 12, 2017 
of fully ionized salts. ${ }^{7}$ These findings indicate that the proper choice of API and its liquid form can influence membrane transport, an important factor in bioavailability. Recently, an in vivo test indicated that the anesthetic effect of lidocaine from Lid. $\mathrm{Ibu}$ is faster and stronger compared with the commercial eutectic mixture of local anesthetics (EMLA, $10 \mathrm{~min}$ vs $1 \mathrm{~h}$ onset time, respectively). The amounts applied to the animals were not reported, but it was inferred by the authors that the concentrations were not comparable (while the Lid.Ibu was applied pure, the commercial mixture contains $2.5 \%$ lidocaine and $2.5 \%$ prilocaine). ${ }^{6}$

Still, one of the unanswered questions is whether this strong dependence of synthetic membrane transport on the interaction between the API and its counterion or hydrogen bond partner would translate to in vivo bioavailability. Due to the complex nature of living systems, an API must first transport across several layers of the epidermis (either through or around cellular membranes) to reach circulatory networks in the dermis and then attain systemic circulation. In the present work, we selected lidocaine-based compounds with different combinations that would affect their degree of ionicity (i.e., salt or hydrogen bonded complex), in order to test our hypothesis that a deep eutectic liquid formulation of lidocaine and ibuprofen (i.e., a liquid cocrystal) would penetrate the skin more effectively than an IL or crystalline salt. The commercially available crystalline salt lidocainium chloride $([\mathrm{Lid}] \mathrm{Cl})$ and the $\mathrm{IL}$ lidocainium docusate $([\mathrm{Lid}][\mathrm{Doc}])$ are salts (in the case of [Lid][Doc], it was found to be $96 \%$ ionized and $4 \%$ complexed $^{9}$ ) (Figure 2), and thus, their

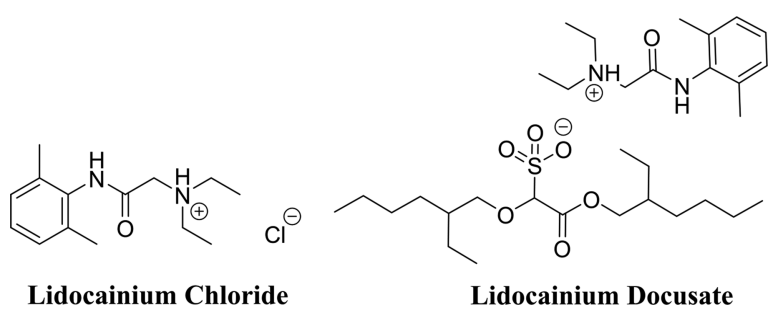

Figure 2. Crystalline lidocanium chloride ([Lid] $] \mathrm{Cl}$; left) and IL lidocainium docusate ([Lid][Doc]; right).

absorption rates are expected to be lower than that of the neutral starting materials. ${ }^{7}$ However, as noted above, the liquid obtained from the 1:1 combination of lidocaine and ibuprofen (Lid-Ibu) was determined to be a closely associated, hydrogen bonded complex, previously defined as a "liquid cocrystal" (Figure 1), ${ }^{10}$ which could more easily permeate the skin as a neutral complex and enter systemic circulation. ${ }^{7}$

The hydrophobic salt [Lid][Doc] and the hydrogen bonded complex Lid.Ibu were synthesized and purified according to previous reports. ${ }^{1,11,12}$ Topical creams of these and the commercially available [Lid] $\mathrm{Cl}$ were then prepared by mixing each compound with an over-the-counter vehicle cream to achieve final concentrations of 3.5 and $5.0 \mathrm{wt} \%$ lidocaine to mimic the commercially available formulations. (It should be noted that, in following the way in which lidocaine concentration is generally reported in the reference cream formulations, i.e., based on weight percent of the entire salt (3.5 or $5.0 \mathrm{wt} \%)$, the molar concentration of lidocaine (and ibuprofen when applied) in each cream varied (Table 1 ) due to the differences in the wt \% of lidocaine in each salt.)

Fourier transform infrared spectroscopy (FT-IR) was used to confirm the presence of the eutectic after loading into the over-
Table 1. Calculated Concentrations of Lidocaine and Ibuprofen in the Applied Creams

\begin{tabular}{|c|c|c|c|c|}
\hline & \multirow[b]{2}{*}{ MW } & \multirow[b]{2}{*}{$\begin{array}{c}\text { compound concentration } \\
\text { (wt \%) }\end{array}$} & \multicolumn{2}{|c|}{$\begin{array}{l}\text { API concentration } \\
(\mathrm{mM})\end{array}$} \\
\hline & & & lidocaine & ibuprofen \\
\hline \multirow[t]{2}{*}[\mathrm{Lid}]{$\mathrm{Cl}$} & 270.80 & 3.5 & 129.2 & $\mathrm{~N} / \mathrm{A}^{a}$ \\
\hline & & 5.0 & 184.6 & N/A \\
\hline \multirow[t]{2}{*}{ Lid·Ibu } & 412.57 & 3.5 & 84.8 & 84.8 \\
\hline & & 5.0 & 121.2 & 121.2 \\
\hline \multirow[t]{2}{*}[\mathrm{Lid}]{$[\mathrm{Doc}]$} & 614.88 & 3.5 & 56.9 & $\mathrm{~N} / \mathrm{A}$ \\
\hline & & 5.0 & 81.3 & N/A \\
\hline
\end{tabular}

the-counter vehicle cream. (The full study is described in the Supporting Information (SI).) Overall, the data confirm that possible interactions with the components of the cream did not result in dissociation of the hydrogen bonded Lid-Ibu eutectic. Interestingly, at Lid.Ibu loadings in the cream vehicle at $10 \mathrm{wt} \%$ or greater, demulsification was observed with conservation of the Lid-Ibu eutectic.

The creams containing the APIs were then applied to a uniform area of the shaved, upper back of adult, male SpragueDawley rats, and blood samples were collected from the tail vain after 30, 60, 120, and $240 \mathrm{~min}$ (see Experimental Procedures for a detailed description of the in vivo pharmacokinetic studies). The levels of API (lidocaine and, when applicable, ibuprofen) were determined in the blood plasma using commercially available ELISA (enzyme-linked immunosorbent assay) kits and quantified using calibration curves (see Experimental Procedures section).

The plasma concentrations of lidocaine observed in our studies (Figure 3) indicate different absorption kinetics depend-

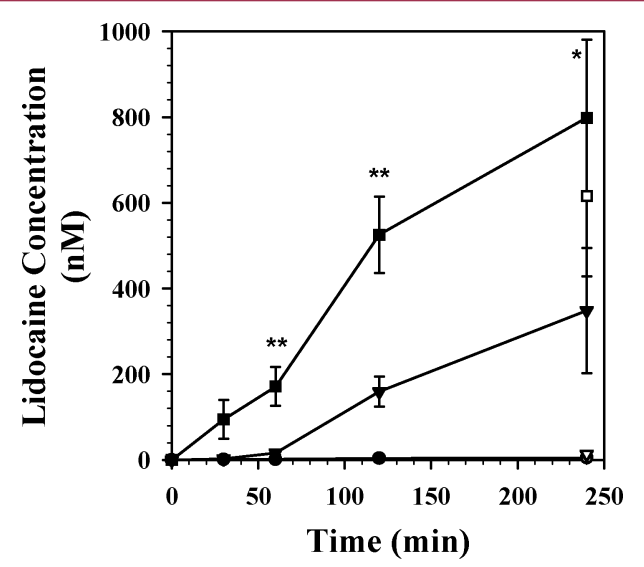

Figure 3. Concentration of lidocaine in blood plasma over time after the topical application of creams containing $5.0 \mathrm{wt} \%$ (filled symbols) and

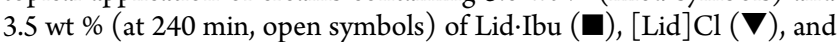
[Lid][Doc] ( $)$. Differences in lidocaine concentrations between treatments at $5.0 \mathrm{wt} \%$ are indicated by double asterisk $(* *)$ if $p<0.05$ or a single asterisk $(*)$ if $p<0.1$.

ing on the lidocaine forms evaluated, confirming that the absorption of an API can be modified through the selection of an appropriate counterion or complexing agent. The differences in the plasma concentrations of lidocaine increased over time (concentrations shown in Table S1, SI). By $120 \mathrm{~min}$, the differences among all three API-containing creams were significant $(p<0.05)$ with the highest concentration observed 
after the application of the neutral complex Lid·Ibu, followed by [Lid]Cl, and finally by [Lid][Doc]. After $240 \mathrm{~min}$, the concentration of lidocaine in the blood plasma continued to increase for Lid.Ibu and [Lid] Cl treated rats, although the treatments began to equilibrate as the rate of absorption started to level off for Lid-Ibu, while [Lid] Cl treated rats began to exhibit greater variability (concentrations shown in Table S1, SI). Still, the differences of lidocaine concentration between $[\mathrm{Lid}] \mathrm{Cl}$ and $\mathrm{Lid} \cdot \mathrm{Ibu}$ or [Lid][Doc] remained significant $(p=0.085$ and $p=$ 0.065 , respectively). Previous reports on lidocaine transdermal absorption in animals indicated that the systematic absorption of the API reaches its maximum after $4 \mathrm{~h}$ from application, when a commercially available lidocaine patch $([\mathrm{Lid}] \mathrm{Cl} 5$ wt $\%$ per patch) was applied to dogs, ${ }^{13}$ or after $12 \mathrm{~h}$ from application, when the patch was applied to cats. ${ }^{14}$

The differences in the plasma concentrations of lidocaine, the area under the curve at $4 \mathrm{~h}$ after treatment $\left(\mathrm{AUC}_{0-4}\right)$, was calculated for the various treatments as an indication of total systemic lidocaine absorption via the trapezoidal rule. ${ }^{15}$ Lidocaine $\mathrm{AUC}_{0-4}$ for $5.0 \mathrm{wt} \%$ [Lid][Doc], [Lid]Cl, or $\mathrm{Lid} \cdot \mathrm{Ibu}$ were found to be 12,602 , or $1763 \mu \mathrm{M} / \mathrm{h}$, respectively. It is interesting to note that, even though the concentration of lidocaine was much higher in the applied [Lid] $\mathrm{Cl}$ (Table 1), the concentration of the API in blood plasma was much lower than $\mathrm{Lid} \cdot \mathrm{Ibu}$, while almost undetectable when [Lid][Doc] was applied. In our previous work, we had reported an increase in lidocaine antinociceptive effect when paired with docusate ([Lid][Doc]) using dimethyl sulfoxide (DMSO) as solvent. ${ }^{11}$ Although the low concentrations of lidocaine in blood plasma and the higher antinociceptive effects might look controversial, the hydrophobicity of the salt and the high molecular weight of the counterion might result in a possible increase in the sorption of the ions while still limiting transport to the blood. In a recent clinical study, when lidocaine was combined with etodolac and applied on Yucatane Micro Pig skin (in vitro test), the membrane transport of the anion through the skin was increased, while lidocaine transport was decreased. ${ }^{16}$ The underlying mechanism has yet to be determined.

The effect of the initial concentration of lidocaine in the applied API-containing creams (i.e., 3.5 vs $5.0 \mathrm{wt} \%$ ) was also studied, based on lidocaine concentrations in blood plasma determined after $240 \mathrm{~min}$ following the application (Figure 3). The concentrations of lidocaine in blood plasma after $240 \mathrm{~min}$ of the application of 3.5 and $5.0 \mathrm{wt} \% \mathrm{Lid} \cdot \mathrm{Ibu}$ in cream indicates no significant differences $(p=0.508)$. This was not true, however, for the creams containing [Lid] Cl, where the lidocaine concentration in blood plasma increases with the applied concentration $(p=0.070)$. However, due to the low concentrations of lidocaine detected after [Lid][Doc] cream application, a comparison of the different absorption after the application of different concentrations of the API-containing creams was not possible (lidocaine concentrations shown in Table S1, SI).

The deep eutectic form Lid-Ibu has previously shown to be transported through a silicon membrane as a complex when dissolved in ethanol. ${ }^{7}$ Therefore, it was expected that the combination of two APIs to form a liquid cocrystal would benefit the systemic sorption of both active ingredients. To demonstrate our hypothesis, we compared the concentrations of lidocaine and ibuprofen in blood plasma after the application of the $5.0 \mathrm{wt} \%$ Lid.Ibu cream (Figure 4). Our results indicate that the two APIs do not reach systemic circulation together and that the ibuprofen reaches circulation much faster and in higher concentrations than

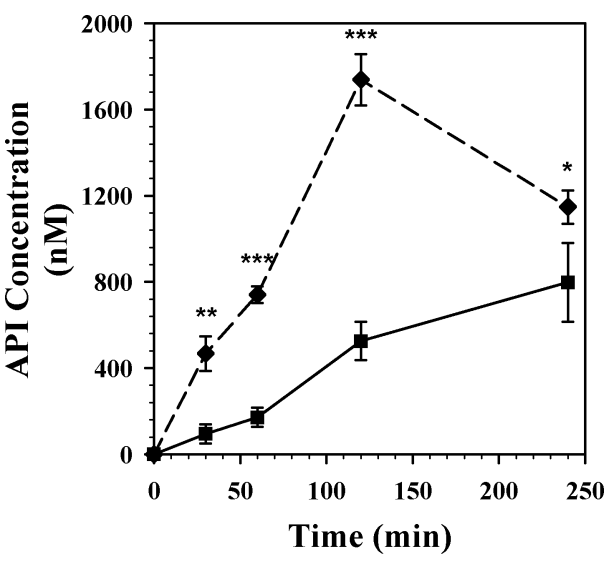

Figure 4. Concentration of lidocaine ( $\mathbf{\square}$, solid line) and ibuprofen ( dashed line) in blood plasma over time after the topical application of creams containing 5.0 wt \% Lid.Ibu. Differences between ibuprofen and lidocaine concentrations are denoted by triple asterisks $(* * *)$ if $p<$ 0.001 , double asterisk $(* *)$ if $p<0.05$, or a single asterisk (*) if $p<0.1$.

lidocaine. The difference in the concentrations between the two APIs increases over time, up to $120 \mathrm{~min}(p<0.0001)$. However, after $240 \mathrm{~min}$ the concentrations of the APIs become similar ( $p=$ 0.110 ), as the ibuprofen concentration in blood plasma starts to decline (ibuprofen concentrations shown in Table S2, SI). Due to the complexity of the matrix of the transdermal absorption mechanism, a fraction of the lidocaine might be complexed with fatty acids and other components present in the skin, thus influencing its absorption and further distribution in tissues and the blood. ${ }^{5,17}$ This and other possibilities related to differences in absorption and/or metabolism of the different APIs will be the subject of further studies.

The effect of the initial concentrations of ibuprofen in the applied creams (3.5 and 5.0 wt \%) on the concentration of the API in blood plasma over time was also examined (Figure 5, ibuprofen concentrations shown in Table S2, SI). Ibuprofen concentrations in blood plasma began to rise, immediately, with slightly elevated absorption observed in the higher treatment even after $30 \mathrm{~min}(p=0.025)$. After $1 \mathrm{~h}$ the rate of absorption of ibuprofen into the blood plasma began to subside for the $3.5 \mathrm{wt} \%$ treatment group, reaching its maximal levels. In contrast, the 5.0

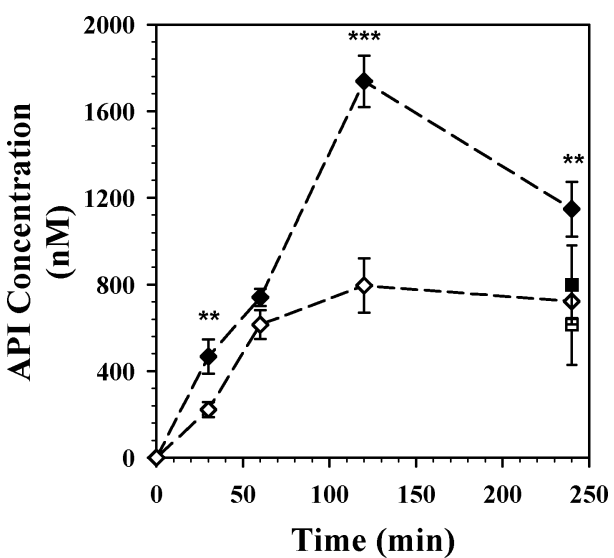

Figure 5. Effect of topical application of 3.5 (open symbols) and 5.0 (filled symbols) wt \% Lid.Ibu on ibuprofen ( ) and lidocaine ( $\boldsymbol{\square})$ concentrations in blood plasma at different times. Differences in ibuprofen concentrations between treatments are indicated by double asterisk $(* *)$ if $p<0.05$ and triple asterisks (***) if $p<0.001$. 
wt \% treatment group continues to absorb a significantly higher quantity of ibuprofen, reaching its maximum level (or $C_{\max }$ ) by 120 min, significantly higher than the 3.5 wt \% treatment at this time point $(p=0.0002)$. The concentration of ibuprofen decreased by $240 \mathrm{~min}$ in the highest treatment group $5.0 \mathrm{wt} \%$ Lid-Ibu $(p=0.004)$, while remaining almost constant for the 3.5 wt \% Lid-Ibu group. Still, even when the blood plasma concentration of ibuprofen began to decline, the difference in concentration between the two groups (rats dosed with $5.0 \mathrm{wt} \%$ vs 3.5 wt $\%$ cream $)$ remained significant $(p=0.008)$. The $\mathrm{AUC}_{0-4}$ for ibuprofen in Lid-Ibu was found to be $2589 \mu \mathrm{M} / \mathrm{h}$ for 3.5 wt $\%$ vs $4643 \mu \mathrm{M} / \mathrm{h}$ for $5.0 \mathrm{wt} \%$, confirming that increased ibuprofen concentration in the cream leads to increased systemic absorption. Interestingly, the differences in the concentrations between the APIs in blood plasma after $240 \mathrm{~min}$ of the application seem to be comparable, independent of the applied concentration (Figure 5), a result that requires further study for a suitable explanation.

In summary, transdermal administration of Lid.Ibu using a vehicle cream resulted in a faster and higher systemic absorption of lidocaine in comparison with the absorption of the commercial salt $[\mathrm{Lid}] \mathrm{Cl}$, suggesting that the unique properties of the liquid cocrystal form might be advantageous for topical application of APIs if systemic absorption is desired. Interestingly, the lidocaine concentrations in blood plasma after the IL [Lid][Doc] was applied were minimal, even lower than those found when [Lid] $\mathrm{Cl}$ was applied, indicating that lidocaine did not reach blood circulation.

The concentrations of lidocaine and ibuprofen in blood plasma after application of Lid-Ibu differed within the evaluating time and the applied concentration, suggesting a differential absorption rate of these APIs, and contradicting our observation in synthetic membrane transport studies. The complex composition of the skin barrier might influence the difference in absorption observed between the different API-liquid forms and the way that these ions are absorbed (forming complexes or interacting with other molecules present in the tissue).

The results presented herein reinforce the importance of selecting proper counterions or complexed forms for APIs which provide tunability to important pharmacokinetic parameters (e.g., bioavailability). Additionally, the data confirm that while there is potential for API-liquid forms to have novel uses that maintain the efficacy of solid formulations, they may not always be more effective. These points should be considered in future experimental designs involving API-liquid forms.

\section{EXPERIMENTAL PROCEDURES}

Chemicals. Pharmaceutical grade (USP) lidocaine and lidocaine hydrochloride were supplied by Spectrum Chemical Mfg. Corp. (Gardena, CA, USA). Methanol (HPLC grade), acetonitrile, chloroform, sodium docusate, and sodium ibuprofen salts (the last two also USP grade), needed for the synthesis of [Lid][Doc] and Lid·Ibu, were purchased from Sigma-Aldrich (St. Louis, MO, USA). Deionized (DI) water was acquired from an in-house system (Culligan Water Systems, Rosemont, IL, USA) with a typical resistivity of $17.4 \mathrm{M} \Omega \cdot \mathrm{cm}$.

The vehicle cream (Lubriderm, fragrance free) was purchased from a local supermarket. The ingredients include aluminum sulfate, calcium acetate, cetyl alcohol, glycerine, glycerylmonostearate SE, methyl paraben, mineral oil, propyl paraben, purified water, sodium hydroxide, sorbitan stearate, stearic acid, stearyl alcohol, and white petrolatum.

Synthesis of the API-Liquid Forms. The API-liquid forms [Lid][Doc] and Lid·Ibu were synthesized and purified according to previous reports (see SI for detailed syntheses). ${ }^{1,1,12}$ Purity ( $>97 \%$ for both synthesized API-liquid forms) and composition of the synthesized compounds was confirmed through NMR spectroscopy $\left({ }^{1} \mathrm{H}\right.$ and ${ }^{13} \mathrm{C}$ NMR). NMR spectra were taken by utilizing a Bruker Avance NMR spectrometer (Karlsruhe, Germany) at $500 \mathrm{MHz}$ for ${ }^{1} \mathrm{H}$ NMR spectroscopy and $125 \mathrm{MHz}$ for ${ }^{13} \mathrm{C}$ NMR spectroscopy.

Preparation of Topical API-Containing Creams. API-liquid forms and $[\mathrm{Lid}] \mathrm{Cl}$ were incorporated into a topical cream as vehicle to mimic available lidocaine preparations and directly compare pharmacokinetics. Creams with different concentrations of [Lid][Doc], Lid. $\mathrm{Ibu}$, and $[\mathrm{Lid}] \mathrm{Cl}(3.5$ and 5.0 wt \%) were prepared by mixing the appropriate amount of these compounds into the control cream and sonication until fully dispersed. These concentrations were selected to match the medium and highest lidocaine-based drug concentration currently available in the commercial products.

In Vivo Pharmacokinetic Studies. Male Sprague-Dawley rats, 610 weeks of age (average weight $240 \mathrm{~g}$ ), were obtained from Charles River Breeding Laboratories (Wilmington, MA, USA) and housed in the AAALAC-approved animal facility at The University of Alabama. Rooms were maintained at $22 \pm 2{ }^{\circ} \mathrm{C}$ with $40-60 \%$ relative humidity and a $12 \mathrm{~h}$ photoperiod. Rats were individually housed in shoe-box type cages with hardwood bedding and given Teklad LM-485 rodent diet (Harlan Laboratories, Indianapolis, IN, USA) and tap water ad libitum. All procedures and protocols performed on the animals were reviewed and approved by The University of Alabama's Institutional Animal Care and Use Committee (IACUC) and were in accordance with established guidelines.

Each animal was weighed and randomly assigned into treatment groups (see Table 2), with $n=8$ animals per treatment group. Control

Table 2. Identification of Treatment Groups; $n=8$ per group

\begin{tabular}{ll} 
group identification & \multicolumn{1}{c}{ treatment } \\
control 1 & sham treatment, no cream \\
control 2 & vehicle control, cream only \\
{$[\mathrm{Lid}] \mathrm{Cl}$ low } & $3.5 \mathrm{wt} \%[\mathrm{Lid}] \mathrm{Cl}$ \\
{$[\mathrm{Lid}] \mathrm{Cl} \mathrm{high}$} & $5.0 \mathrm{wt} \%[\mathrm{Lid}] \mathrm{Cl}$ \\
{$[\mathrm{Lid}][\mathrm{Doc}]$ low } & $3.5 \mathrm{wt} \%[\mathrm{Lid}][\mathrm{Doc}]$ \\
{$[\mathrm{Lid}][\mathrm{Doc}]$ high } & $5.0 \mathrm{wt} \%[\mathrm{Lid}][\mathrm{Doc}]$ \\
$\mathrm{Lid} \cdot \mathrm{Ibu}$ low & $3.5 \mathrm{wt} \% \mathrm{Lid} \cdot \mathrm{Ibu}$ \\
Lid·Ibu high & $5.0 \mathrm{wt} \% \mathrm{Lid} \cdot \mathrm{Ibu}$
\end{tabular}

groups were an untreated sham-operated group and the vehicle control (cream only). Twenty-four hours prior to treatment, the rats were shaved with veterinarian-provided electric animal clippers on the upper back (between the shoulder blades). To reduce stress and ease of handling, prior to shaving, the rats were placed under anesthesia using isoflurane. At the time of treatment, the rats were anesthetized, an initial blood sample was collected $(0 \mathrm{~h})$, and the appropriate treatment cream was applied to a consistent square area and covered with sterile gauze. A square template was used on each rat to ensure consistent surface area between rats. Doses applied were calculated based on body mass of the animals. For example, a $250 \mathrm{~g}$ rat would receive $0.25 \mathrm{~g}$ of cream in the specified area. To reduce the stress of handling, the rats were placed in individual plastic holding apparatus (544-RR, Rat Restrainer, PLASLABS) that allowed for easy access to the tail vein for blood collection. Prior to experiments, several nonstudy rats were placed in the plastic holders for the entire length of the experiment $(>240 \mathrm{~min})$ and monitored for increased heart rate, labored breathing, and other signs of distress. No signs of stress were observed by placement in the plastic holders for upward of $4 \mathrm{~h}$.

Blood samples were collected 30,60, 120, and $240 \mathrm{~min}$ after the application of the test cream in anticoagulant (ethylenediaminetetraacetic acid, EDTA) coated capillary tubes to monitor levels of API in the blood plasma. While ibuprofen was determined for the entire period (from 0 to $240 \mathrm{~min}$ ) at both concentrations, lidocaine was determined within the entire period at $5.0 \mathrm{wt} \%$, but only after $240 \mathrm{~min}$ at $3.5 \mathrm{wt} \%$. Samples were centrifuged immediately after collection and maintained at $-80{ }^{\circ} \mathrm{C}$ until analysis. Following the final blood collection, animals were euthanized by $\mathrm{CO}_{2}$ asphyxiation in accordance with institutional guidelines and the AVMA Guidelines for the Euthanasia of Animals. ${ }^{18}$ 
Quantification of Lidocaine and Ibuprofen. Plasma lidocaine and ibuprofen levels were measured in blood plasma using commercially available ELISA kits (Neogen Corporations, Lexington, KY, USA). Blood plasma samples were allowed to thaw on ice, then pipetted directly into the appropriate well plate in duplicate. Standards were prepared by introducing known concentrations of lidocaine or ibuprofen into untreated (blank) rat plasma to obtain calibration curves (described below). All determinations were performed in duplicate. The protocol supplied with the ELISA kit was followed without modifications. Absorbance was measured at $670 \mathrm{~nm}$ using a Synergy 2 Multi-Mode Reader (BioTek Instruments, Inc., Winooski, VT, USA).

Calibration curves were calculated in the range $21.4-2133.6 \mathrm{nM}$ (5$500 \mathrm{ng} / \mathrm{mL})$ for lidocaine and $24.2-3635.6 \mathrm{nM}(5-750 \mathrm{ng} / \mathrm{mL})$ for ibuprofen. Standard samples were processed in the same way as test samples. Data were plotted as logit $\left(B / B_{0}\right)$ vs log concentration, and a linear regression fit was performed. Logit $\left(B / B_{0}\right)$ was calculated as

$$
\operatorname{logit}\left(\frac{B}{B_{0}}\right)=\ln \left[\left(\frac{B / B_{0}}{1-B / B_{0}}\right)\right]
$$

with $\mathrm{B} / \mathrm{B}_{0}$ was calculated as

$$
\left(\frac{B}{B_{0}}\right)=\left(\frac{\text { standard absorbance }- \text { NSB }}{\text { average } B_{0} \text { absorbance }- \text { NSB }}\right)
$$

where $B_{0}$ is the absorbance obtained when binding is maximum, and NSB absorbance is obtained from nonspecific binding.

$\mathrm{AUC}_{0-4}$ was determined using the linear trapezoidal rule by calculating the sum of individual trapezoids following treatment, which was calculated as follows:

$$
\mathrm{AUC}_{t_{1}-t_{2}}=\left(\frac{\frac{1}{2} C_{1}+C_{2}}{t_{2}-t_{1}}\right)
$$

where $C$ is the concentration at the time point, $t$ (in hours), for API blood plasma measurements.

Statistical Analyses. Normally distributed data, reported as mean \pm standard error of the mean, was statistically analyzed by Student's $t$ test or one-way ANOVA, using the software IBM SPSS Statistics Version 23.0.0.0 (2011) (IBM, Armonk, NY, USA) and R statistical software version 3.2.1 (R Foundation for Statistical Computing, Vienna, Austria). Before the statistical analysis, the Shapiro-Wilk test was used to verify that the samples come from a normal distribution. Once this requirement was passed, a Levene test was used to confirm the homogeneity of variances. If any (or both) of these requirements was not fulfilled by the group of samples, data was transformed (using ln or sin functions) until the requirements agreed. Before performing the test, a "significance level" (or test's threshold value, $\alpha$ ) of 0.05 was chosen, followed by calculation of a function of the observed sample results ( $p$ value), and the null hypothesis was tested at the chosen $\alpha$-level of 0.05 . The data were then subjected to Student's $t$ test or one-way ANOVA followed by Tukey's HSD (Honest Significant Difference) test for post hoc comparisons when needed. Statistical significance was considered when $p<0.05$.

\section{ASSOCIATED CONTENT}

\section{(S Supporting Information}

The Supporting Information is available free of charge on the ACS Publications website at DOI: 10.1021/acsmedchemlett.6b00504.

Lidocaine concentration in blood; synthesis of lidocaine ibuprofen and lidocainium docusate; statistical analyses; determination of Lid.Ibu eutectic form in the cream (PDF)

\section{AUTHOR INFORMATION}

\section{Corresponding Author}

*E-mail: robin.rogers@mcgill.ca.

ORCID

Robin D. Rogers: 0000-0001-9843-7494

Present Address

${ }^{\#}$ K.R.D.B.: Department of Molecular Biology, University of Wyoming, Laramie, Wyoming 82071, United States.

\section{Author Contributions}

The manuscript was written through contributions of all authors. All authors have given approval to the final version of the manuscript.

\section{Notes}

The authors declare the following competing financial interest(s): The authors declare the following competing financial interest(s): Dr. Robin D. Rogers and Dr. G. Gurau are inventors on related patents and have partial ownership of 525 Solutions, Inc. Dr. J. L. Shamshina is an inventor on related patents and is an employee of 525 Solutions, Inc. The University of Alabama and McGill University maintain approved Conflict of Interest Management Plans.

\section{ACKNOWLEDGMENTS}

This work was supported in part by a Howard Hughes Medical Institute (HHMI) Undergraduate Biological Sciences Program grant to The University of Alabama. This research was undertaken, in part, thanks to funding from the Canada Excellence Research Chairs Program.

\section{ABBREVIATIONS}

API-ILs, active pharmaceutical ingredient-ionic liquids; IL, ionic liquid; [Lid]Cl, lidocainium chloride; [Lid][Doc], lidocainium docusate; Lid·Ibu, lidocaine ibuprofen

\section{REFERENCES}

(1) Rogers, R. D.; Daly, D. T.; Swatloski, R. P.; Hough, W. L.; Davis, J. H., Jr.; Smiglak, M.; Pernak, J.; Spear, S. K. Multi-Functional Ionic Liquid Compositions for Overcoming Polymorphism and Imparting Improved Properties for Active Pharmaceutical, Biological, Nutritional, and Energetic Ingredients. US8232265 (2012); PCT/US2006/039454; WO 2007/044693 A2 (2007); MX 301158 (2012); CN 200680046195.7 (2013).

(2) Hough, W. L.; Rogers, R. D. Ionic Liquids Then and Now: From Solvents to Materials to Active Pharmaceutical Ingredients. Bull. Chem. Soc. Jpn. 2007, 80, 2262-2269.

(3) Kelley, S. P.; Narita, A.; Holbrey, J. D.; Green, K. D.; Reichert, W. M.; Rogers, R. D. Understanding the Effects of Ionicity in Salts, Solvates, Co-Crystals, Ionic Co-Crystals, and Ionic Liquids, Rather than Nomenclature, Is Critical to Understanding Their Behavior. Cryst. Growth Des. 2013, 13, 965-975.

(4) Bica, K.; Rogers, R. D. Confused Ionic Liquid Ions - A "Liquification" and Dosage Strategy for Pharmaceutically Active Salts. Chem. Commun. 2010, 46, 1215-1217.

(5) Bica, K.; Shamshina, J. L.; Hough, W. L.; MacFarlane, D. R.; Rogers, R. D. Liquid Forms of Pharmaceutical Co-crystals: Exploring the Boundaries of Salt Formation. Chem. Commun. 2011, 47, 22672269.

(6) Park, H. J.; Prausnitz, M. R. Lidocaine-Ibuprofen Ionic Liquid for Dermal Anesthesia. AIChE J. 2015, 61, 2732-2738.

(7) Wang, H.; Gurau, G.; Shamshina, J. L.; Cojocaru, O. A.; Janikowski, J.; MacFarlane, D. R.; Davis, J. H., Jr.; Rogers, R. D. Simultaneous Membrane Transport of Two Active Pharmaceutical Ingredients by Charge Assisted Hydrogen Bond Complex Formation. Chem. Sci. 2014, 5, 3449-3456. 
(8) Palmer, M. Biochem. Pharmacol.; John Wiley \& Sons: Hoboken, 2012.

(9) Moreira, D. N.; Fresno, N.; Pérez-Fernández, R.; Frizzo, C. P.; Goya, P.; Marco, C.; Martins, M. A. P.; Elguero, J. Brønsted Acid-Base Pairs of Drugs As Dual Ionic Liquids: Nmr Ionicity Studies. Tetrahedron 2015, 71, 676-685.

(10) Abbott, A. P.; Boothby, D.; Capper, G.; Davies, D. L.; Rasheed, R. K. Deep Eutectic Solvents Formed between Choline Chloride and Carboxylic Acids: Versatile Alternatives to Ionic Liquids. J. Am. Chem. Soc. 2004, 126, 9142-9147.

(11) Hough, W. L.; Smiglak, M.; Rodríguez, H.; Swatloski, R. P.; Spear, S. K.; Daly, D. T.; Pernak, J.; Grisel, J. E.; Carliss, R. D.; Soutullo, M. D.; Davis, J. H.; Rogers, R. D. The Third Evolution of Ionic Liquids: Active Pharmaceutical Ingredients. New J. Chem. 2007, 31, 1429-1436.

(12) Bica, K.; Rodríguez, H.; Gurau, G.; Cojocaru, O. A.; Riisager, A.; Fehrmann, R.; Rogers, R. D. Pharmaceutically Active Ionic Liquids with Solids Handling, Enhanced Thermal Stability, and Fast Release. Chem. Commun. 2012, 48, 5422-5424.

(13) Weiland, L.; Croubels, S.; Baert, K.; Polis, I.; De Backer, P.; Gasthuys, F. Pharmacokinetics of a Lidocaine Patch $5 \%$ in Dogs. J. Vet. Med., A 2006, 53, 34-39.

(14) Ko, J. C. H.; Maxwell, L. K.; Abbo, L. A.; Weil, A. B. Pharmacokinetics of lidocaine following the application of $5 \%$ Lidocaine Patched to Cats. J. Vet. Pharmacol. Ther. 2008, 31, 359-367.

(15) Kwon, Y. Handbook of Essential Pharmacokinetics, Pharmacodynamics, and Drug Metabolism for Industrial Scientists; Kluwer Academic/ Plemun Publishers: New York, 2001.

(16) Miwa, Y.; Hamamoto, H.; Ishida, T. Lidocaine Self-Sacrificially Improves the Skin Permeation of the Acidic and Poorly Water-Soluble Drug Etodolac via Its Transformation into an Ionic Liquid. Eur. J. Pharm. Biopharm. 2016, 102, 92-100.

(17) Griffin, P. J.; Cosby, T.; Holt, A. P.; Benson, R. S.; Sangoro, J. R. Charge Transport and Structural Dynamics in Carboxylic-Acid-Based Deep Eutectic Mixtures. J. Phys. Chem. B 2014, 118, 9378-9385.

(18) AVMA - American Veterinary Medical Association. AVMA Guidelines for the Euthanasia of Animals: 2013 Edition. https://www. avma.org/KB/Policies/Pages/Euthanasia-Guidelines.aspx (accessed December 11, 2016). 